\title{
12. Chromosome Study of Acatalasemia
}

\author{
By Koji OHKura, ${ }^{*}$ Hiroko NaKagawa, ${ }^{*)}$ Naoya KosaKa, ${ }^{* *)}$ \\ Masanori SADAMOTO, ${ }^{* *}$ and Shigeo TAKAHARA**) \\ (Comm. by Tanemoto. FuruhatA, M.J.A., Jan. 13, 1964)
}

Acatalasemia is a congenital biochemical abnormality characterized by the lack of enzyme catalase in the human blood cells (S. Takahara, 1947 and latest summarized paper 1963). In this trait, heterozygotic state for the gene corresponding to acatalasemia is identified from both homozygotic states by biochemical examinations.

This paper is concerned with the chromosome study of culture cells of skin and leucocytes from acatalasemic and hypocatalasemic individuals.

Cases. Case 1. (J. F., Acatalasemia, male, 30 years old.) Skin specimens were obtained from his forearm by surgical technique. The specimens were cultured and the 24th and 27th generations (108 and 117 days) of subcultured materials were examined.

Case 2. (H. W., Acatalasemia, Female, 29 years old.) She suffered from progressive oral gangrene about 20 years ago, and was admitted to the Dental Hospital of the Tokyo Medical and Dental University for plastic surgery. Skin specimens were obtained and cultured. In this case, the chromosomes were examined in the cells of the short term culture materials of skin and blood. The chromosomes of the subcultured materials of skin at the 5th generation (19 days) were also examined.

Case 3. (M. T., Acatalasemia, Female, 28 years old.) This case has almost the same history as that of case 2 , and the skin specimens were obtained from the forearm when she received a plastic operation at the Osaka Dental College Hospital. The 9th generation (46 days) of subcultured material of the skin was examined.

Case 4. (K. T., Acatalasemia, Female, 25 years old.) She has no history of oral gangrene. Blood specimens were obtained and cultured.

Case 5. (K. F., Hypocatalasemia, Female, 51 years old.) She is the mother of Case 1. Skin specimens were obtained and cultured. The 20th generation (90 days) of subcultured materials was examined.

Case 6. (S. A., Normal, 22 years old.) Skin specimens were obtained and the 20th generation (90 days) of subcultured materials was

This study was supported in part by the Research Training Grant (2G-664 and 2G$664 \mathrm{C1}$ ) and the Research Grant (AM 06793-01 GEN) from the National Institutes of Health, the U. S. Public Health Service.

*) Department of Human Genetics, Tokyo Medical and Dental University.

**) Department of Otorhinolaryngology, Okayama University Medical School. 
examined as a control.

Case 7. (Y. I., Normal, Female, 22 years old.) Blood specimens were studied in the short term culture of leucocytes as a control.

Tissue Culture and Cytological Techniques. Tissue culture techniques and cytological techniques used in this study were briefly summarized as follows:

Peripheral blood culture

1) Obtain about $10 \mathrm{cc}$ of heparinized blood.

2) Add $0.25 \mathrm{cc}$ Phytohemagglutinin (Difco).

3) Separate the serum containing the leucocytes.

4) Mix $2 \mathrm{cc}$ of the serum and $8 \mathrm{cc}$ of TC 199 (added penicillin. and streptomycin).

5) Incubate at $37^{\circ} \mathrm{C}$ for $3 \sim 4$ days.

6) Add colchicine and incubate for 4 hours.

7) Treat with hypotonic solution.

8) Collect the leucocytes.

Skin Culture

1) Explant the pieces of skin on the cover slip with plasma clot..

2) Medium $\left\{\begin{array}{l}2 \text { parts of human serum } \\ 2 \text { parts of chick embryo extract } \\ 6 \text { parts of TC } 199 .\end{array}\right.$

3) Incubate at $37^{\circ} \mathrm{C}$. Change two thirds of the medium twice: a week.

4) After 2 weeks, subculture is begun.

5) Medium for subculture is the same as mentioned above.

6) Colchicine was used for accumulation of mitotic cells.

Fixation. As a fixative, Carnoy's mixture (3 volumes of absolute alcohol and 1 volume of glacial acetic acid) was used.

Staining. Most of slides were made by air dry method; the squash method was also used. Slides were stained with acetic Dahlia, Feulgen stain, and acetic Orcein.

Cytological Observations. All types of preparations were studied.

Table

\begin{tabular}{llrrl}
\hline Case Diagnosis & Sex & $<4454647$ & \multicolumn{1}{c}{ Tissue } \\
\hline 1 Acat. & M & 121 & Skin, Subculture 108 and 117 days \\
2 Acat. & F & 29 & Leucocytes, Short term culture \\
& & 35 & Skin, Short term culture \\
3 Acat. & F & 23 & Skin, Subculture 14 days \\
4 Acat. & F & 25 & Skin, Subculture 46 days \\
5 Hypocat. & F & 1 & 30 & Leucocytes, Short term culture \\
6 Normal & F & 149 & Skin, Subculture 90 days \\
7 Normal & F & 45 & Leucocytes, Short term culture \\
\hline
\end{tabular}


by microscopic examination using oil immersion and individual chromosomes of cells were sketched. Then, some microphotographs were taken and individual chromosomes for final matching were cut out from enlargements.

Some 20 to 50 cells were examined and the number of chromosomes in each material was counted. These results were shown in Table.

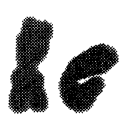

I

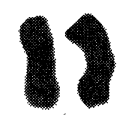

2

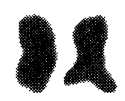

3

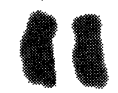

$4-5$

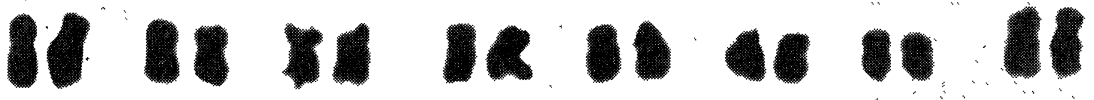 $6-12$
1010
$13-15$
$16-18$

\section{e 1}
$19-20$
- - $\bullet$
$21-22$

4018

Fig. 1. Karyotype of acatalasemia, leucocytes.
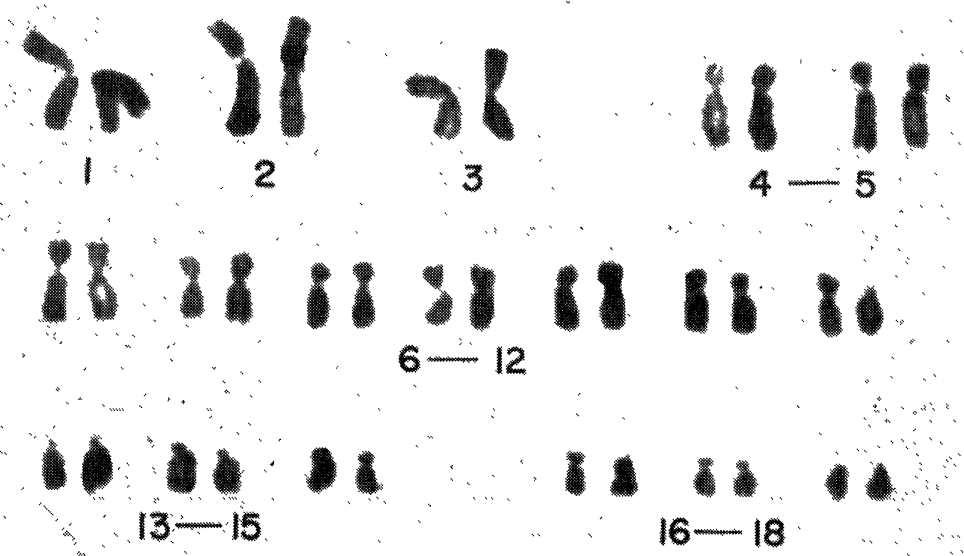

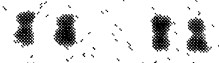

$19-20$

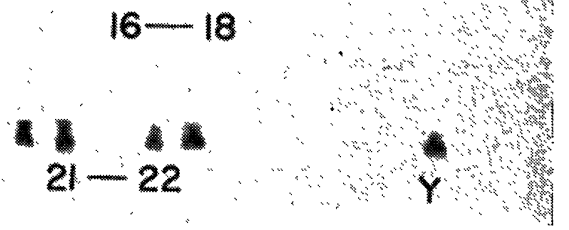

Fig. 2. Karyotype of acatalasemia, skin culture. 


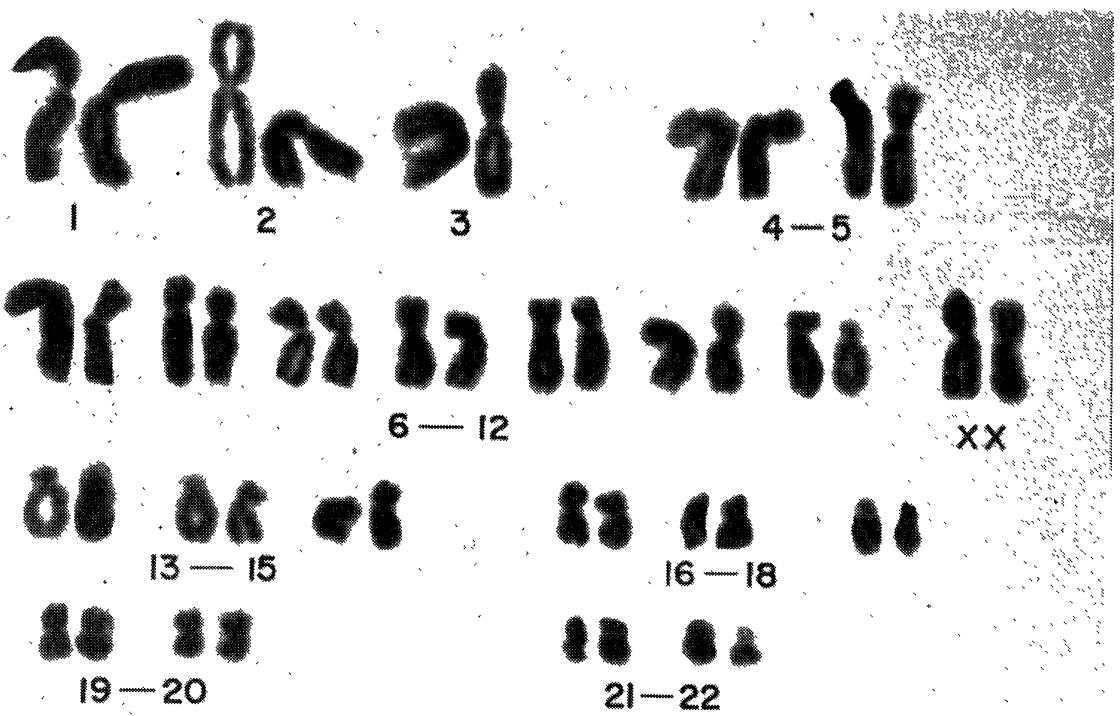

Fig. 3. Karyotype of hypocatalasemia, skin culture.

Five karyotypes of each material were analyzed, but no abnormality was found (Figs. 1-3).

Conclusion. In this study, no chromosome aberration was found in the cases of acatalasemia and hypocatalasemia: Acatalasemia is transmitted as an autosomal recessive trait.

Acknowledgement. The authors are indebted to Dr. T. Shiroyama, of Osaka Dental College, and Dr. H. Ito and Dr. M. Kawamura, of Tokyo Medical and Dental University, for their kindness in obtaining the materials. We also express our thanks to Mr. Y. Kuroda and Miss Y. Ishiodori for their technical assistances.

\section{Reference}

Takahara, S.: Acatalasemia. Jap. Jour. Human Genet., 7, 37-59 (1963). 